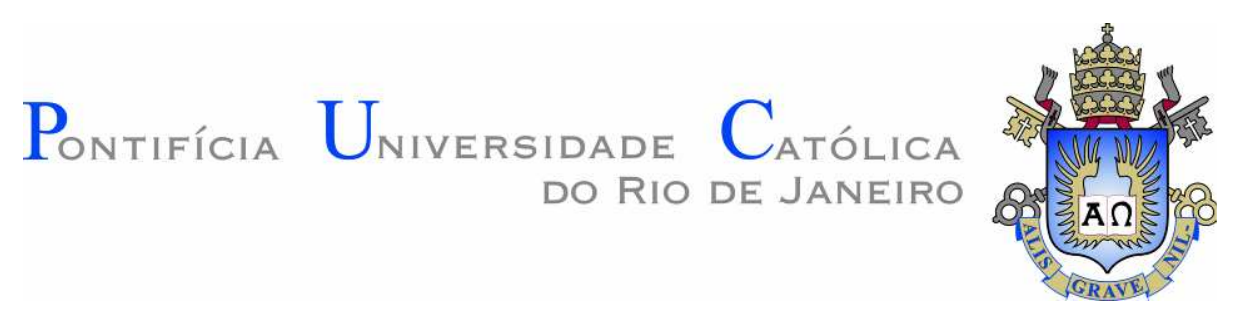

Marcel Passos Zylberberg

\begin{abstract}
Análise da transmissão de impacto de diferentes calçados militares
\end{abstract}

Dissertação de Mestrado

Dissertação apresentada como requisito parcial para obtenção do grau de Mestre pelo Programa de Pós-Graduação em Engenharia de Produção do Departamento de Engenharia Industrial da PUC-Rio.

Orientadora: Prof. Fernanda Maria Pereira Raupp

Rio de Janeiro

Fevereiro de 2012 


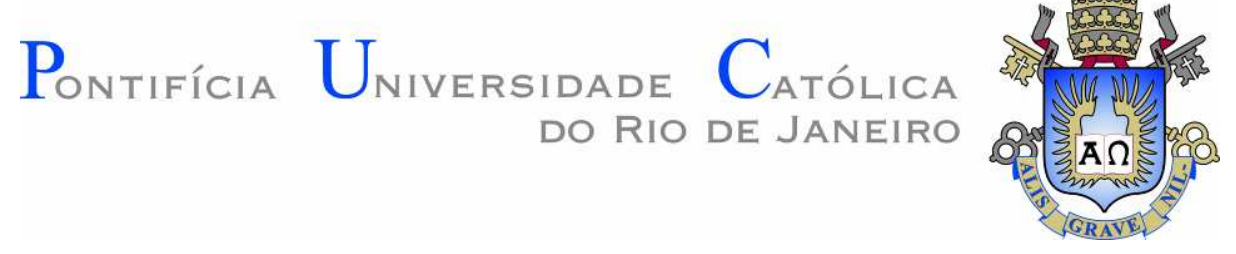

Marcel Passos Zylberberg

\section{Análise da transmissão de impacto de diferentes calçados militares}

Dissertação apresentada como requisito parcial para obtenção do grau de Mestre pelo Programa de Pós-Graduação em Engenharia de Produção do Departamento de Engenharia Industrial da PUC-Rio. Aprovada pela Comissão Examinadora abaixo assinada.

Prof. Fernanda Maria Pereira Raupp

Orientadora

Departamento de Engenharia Industrial - PUC - Rio

Prof. Liliam Fernandes de Oliveira Universidade Federal do Rio de Janeiro - UFRJ

Prof. Adriane Mara de Souza Muniz Escola de Educação Física do Exército - EsEFEx

Prof. Roger Gomes Tavares de Mello Escola Naval - EN

Prof. José Eugênio Leal Coordenador Setorial do Centro Técnico Científico - PUC - Rio 
Todos os direitos reservados. É proibida a reprodução total ou parcial do trabalho sem autorização do autor, do orientador e da universidade.

\section{Marcel Passos Zylberberg}

Graduou-se em Ciências Militares na AMAN (Academia Militar das Agulhas Negras), e durante os dois anos que trabalhou como oficial em Campo Grande - MS, cursou três semestres da graduação em Educação Física na UFMS (Universidade Federal do Mato Grosso do Sul). Após optar por nova área de trabalho, mudou-se para o Rio de Janeiro e graduou-se em Engenharia de Materiais no IME (Instituto Militar de Engenharia). Trabalhou durante três anos no Arsenal de Guerra do Rio, período no qual cursou uma Pós-graduação em Conhecimentos Militares. Ingressou em seguida no Programa de Pós-graduação em Engenharia de Produção do Departamento de Engenharia Industrial da PUC-Rio para obtenção do título de Mestre. Nesse período foi transferido para o IPCFEx (Instituto de Pesquisa da Capacitação Física do Exército), onde exerce a função de pesquisador do Laboratório de Biomecânica. Participou de diversos congressos nas áreas de ergonomia, engenharia e biomecânica.

Ficha Catalográfica

Zylberberg, Marcel Passos

Análise da transmissão de impacto de diferentes de calçados militares / Marcel Passos Zylberberg; orientadora: Fernanda Maria Pereira Raupp. - 2012.

81 f. : il. (color.) ; $30 \mathrm{~cm}$

Dissertação (mestrado)-Pontifícia Universidade Católica do Rio de Janeiro, Departamento de Engenharia Industrial, 2012.

Inclui bibliografia

1. Engenharia Industrial - Teses. 2. Ergonomia. 3. Biomecânica. 4. Análise Cinemática. 5. Acelerômetros. 6. Calçados militares. I. Raupp, Fernanda Maria Pereira. II. Pontifícia Universidade Católica do Rio de Janeiro. Departamento de Engenharia Industrial. III. Título. 


\section{Agradecimentos}

Agradeço primeiramente a Força Maior que faz passar os segundos e dita o ritmo dos acontecimentos na vida. Obrigado por ter proporcionado as oportunidades de estudo e aperfeiçoamento que tive até hoje. À minha família, que me inseriu e deu apoio neste mundo, e mesmo à distância se fez sempre presente, me sustentando em todos os momentos.

Às pessoas que estiveram mais próximas nesse período e participaram de alguma forma nesta dissertação ou possibilitaram a execução da mesma. Obrigado aos amigos Adriane, Beatriz, Fernanda, Jaqueline, Lílian, Priscila, Márcio Leão, Renault, Roger, Izabela, Guilherme e Natália.

À minha Orientadora Fernanda Raupp, pela compreensão, apoio incondicional e acompanhamento nessa etapa. Aos professores que participaram da Comissão Examinadora.

À pessoa do Gen Ribeiro Souto, pela oportunidade da minha inclusão no convênio do CTEx com a PUC-Rio, e conseqüente realização deste curso de mestrado. Ao IPCFEx pela oportunidade de realizar os testes. Aos voluntários que participaram do estudo, sem os quais esta pesquisa experimental não seria possível.

Aos professores, amigos e funcionários do DEI da PUC-Rio, pelos ensinamentos e apoio proporcionados, sendo o professor Dalcol, a Cláudia, a Ana e o Eduardo àqueles os quais dei mais trabalho.

Não fossem os conhecimentos adquiridos pelas disciplinas estudadas, o apoio e ensinamento de amigos da PUC e companheiros do trabalho, as críticas construtivas sobre elaboração da dissertação, e os momentos de descontração nessa cidade do Rio de Janeiro, este trabalho não seria concluído. Guardarei sempre com muita estima as lembranças desse período de 2009 a 2011. Agradeço a todos que me ajudaram nestes três anos para conseguir concluir este curso. 


\section{Resumo}

Zylberberg, Marcel Passos; Raupp, Fernanda Maria Pereira (Orientadora). Análise da transmissão de impacto de diferentes calçados militares. Rio de Janeiro, 2012. 81p. Dissertação de Mestrado - Departamento de Engenharia Industrial, Pontifícia Universidade Católica do Rio de Janeiro.

A presente dissertação teve como objetivo analisar a transmissão de impacto de três diferentes modelos de um calçado militar por meio de sua influência na aceleração tibial. A variável pico de aceleração e as intensidades da frequência do sinal em determinadas faixas foram medidas no eixo vertical, por intermédio da técnica de acelerometria, visando caracterizar os coturnos quanto sua adequação ergonômica e biomecânica como ferramenta de trabalho para os militares. Dez (10) sujeitos caminharam numa esteira ergométrica em velocidade constante e padronizada, descalços e com três calçados diferentes. As variáveis foram medidas por meio de um acelerômetro capacitivo fixado sobre a pele, na tíbia, e o procedimento reavaliado em dois dias diferentes. Os valores encontrados apresentaram excelente confiabilidade. $\mathrm{O}$ valor médio de redução do pico de impacto mensurado na tíbia dos sujeitos utilizando os coturnos foi próximo de $50 \%$, em relação à situação descalço. Após a análise da média de pico da aceleração, os resultados encontrados não foram conclusivos quanto à diferença significativa de absorção de impacto entre os tipos de coturno, porém em conjunto com as avaliações de intensidades de faixas de frequência da transmissão do impacto, parece que o coturno comum apresentou melhor resultado. Existe a necessidade de novos estudos com outros equipamentos, como palmilhas instrumentadas e plataformas de força, para caracterização mais precisa dos calçados, bem como realizar testes de reavaliação de propriedades mecânicas após ciclos de desgastes dos calçados.

\section{Palavras-chave}

Ergonomia; biomecânica; análise cinemática, acelerômetros; calçados militares. 


\section{Abstract}

Zylberberg, Marcel Passos; Raupp, Fernanda Maria Pereira (Advisor). Analysis of the impact transmitted by different military boots. Rio de Janeiro, 2012. 81p. MSc. Dissertation - Departamento de Engenharia Industrial, Pontifícia Universidade Católica do Rio de Janeiro.

The dissertation aimed to analyze the transmission of impact of three different models of a military boot through its influence on tibial acceleration. The variable peak acceleration, and the intensities of the signal in certain frequency bands, were measured on the vertical axis using accelerometer, in order to characterize the boots suitability as ergonomic and biomechanics tool for the military. Ten (10) subjects walked on a treadmill at constant and standard speed, barefoot and with three different shoes. The variables were measured using a capacitive accelerometer fixed to the skin on the tibia and the procedure reevaluated on two different days. The values found showed excellent reliability. The average reduction in peak impact measured in the tibia of the subjects using the boots was near 50\%, as compared to the barefoot. After analysis of the average peak acceleration, the results were not conclusive as to the significant difference in shock absorption between the types of boots. Studying the acceleration data in conjunction with assessments of intensities of frequency bands of the transmission of impact, it seems that the common buskin had the best result. There is a need for further studies with other equipment, such as instrumented shoe insoles and force platforms, for more precise characterization of the military boots as well as testing of mechanical properties after shoe degradation cycles.

\section{Keywords}

Ergonomics; biomechanics; kinematic analysis; accelerometers; military boots. 
Sumário

1. INTRODUÇÃO 12

2. FUNDAMENTAÇÃO TEÓRICA 16

2.1. PARTICULARIDADES MILITARES 16

2.2. ERGONOMIA FÍSICA 23

2.3. BIOMECÂNICA 28

2.3.1. ACELEROMETRIA 30

2.3.2. ANÁLISE DE MARCHA 36

2.3.3. CINEMETRIA 39

3. MATERIAIS E MÉTODOS 41

3.1. AMOSTRA 41

3.2. CALÇADOS MILITARES

3.3. EQUIPAMENTOS

3.4. PROTOCOLO EXPERIMENTAL 47

3.5. ANÁLISE E PROCESSAMENTO DOS SINAIS 49

4. APRESENTAÇÃO E ANÁLISE DOS RESULTADOS 52

4.1. DADOS DE CINEMETRIA 52

4.2. ACELEROMETRIA E TRANSMISSÃO DE IMPACTO 56

5. CONCLUSÃO 69

REFERÊNCIAS BIBLIOGRÁFICAS

APÊNDICE A

APÊNDICE B $\quad 75$

APÊNDICE C $\quad 78$

$\begin{array}{ll}\text { ANEXO A } & 81\end{array}$ 


\section{Lista de Figuras}

Figura 1. Coturnos da 2 ${ }^{\mathrm{a}} \mathrm{GM}(\mathrm{a})$, Exército Brasileiro (b) e Exército Americano (c)

Figura 2. Cronograma de Instrução de um Batalhão de Infantaria

Figura 3. Termos anatômicos para descrever posição e direção (Godfrey, 2008)

Figura 4. Sistema de coordenadas para análise de movimento (Winter, 1990:13) 30

Figura 5. Esquema representativo do acelerômetro capacitivo 32

Figura 6. llustração da montagem com BMA na tíbia (adaptado de Hwang, 2006) 33

Figura 7. Fases da marcha (Perry, 2005:3) 37

Figura 8. Contato com o solo abrupto (Perry, 2005:35) 38

Figura 9. Coturnos avaliados - CC (a), CE (b), CM (c) 42

Figura 10. Representação esquemática do solado (InstrNorm/DAbst EB) 43

Figura 11. Acelerômetro fixado sobre a tuberosidade da tíbia 45

Figura 12. Acelerômetro MMA7361 Sparkfun - instrumentado 45

Figura 13. Equipamento de análise cinemática 3D 46

Figura 14. Localização dos marcadores passivos da cinemetria 48

Figura 15. Picos de acelerometria considerados para cálculo da média 51

Figura 16. PSD para a cinemetria e aceleração indicando mesma frequência máxima

Figura 17. Gráfico de FVA e acelerometria - falta de sincronia ao longo das amostras

Figura 18. Gráficos da média do pico de aceleração dos testes 58

Figura 19. Gráfico representando as bandas de frequência avaliadas 59

Figura 20. Gráfico Box-Plot da variável média do pico de aceleração 61

Figura 21. Coturnos CC (a), CE (b) e CM (c) seccionados - estrutura dos solados

62

Figura 22. Gráfico da magnitude média das bandas de frequência avaliadas

64

Figura 23. Gráfico Box-Plot da variável magnitude das bandas de frequência

65

Figura 24. Equações de predição dos valores de pico de aceleração

Figura 25. Gráfico de probabilidade normal dos resíduos studentized

Figura 26. Gráfico de resíduos studentized por ordem de teste 


\section{Lista de tabelas}

Tabela 1. Dados técnicos sobre materiais e dimensões dos diferentes modelos de coturnos

Tabela 2. Valores médios e extremos de pico de aceleração dos testes 56

Tabela 3. Reprodutibilidade intra-avaliações das variáveis de aceleração

60

Tabela 4. Média \pm DP dos picos de aceleração e testes estatísticos

Tabela 5. Média \pm DP da potência do sinal nas diferentes bandas de frequência e testes estatísticos 


\section{Lista de abreviaturas}

EsEFEx - Escola de Educação Física do Exército

EN - Escola Naval

EB - Exército Brasileiro

IME -Instituto Militar de Engenharia

AMAN - Academia Militar das Agulhas Negras

RUE - Regulamento de Uniformes do Exército

OM - Organização Militar

$\mathrm{CC}$ - coturno de lona preto comum

$\mathrm{CE}$ - coturno de lona preto especial

$\mathrm{CM}$ - coturno marrom

GM - Guerra Mundial

$\mathrm{kJ}$ - quilojoules

BMA - Acelerômetros Montados nos Ossos

SMA - Acelerômetros Montados na Pele

RMS - raiz média quadrática

FFT -Transformada rápida de Fourier

FM - frequência máxima

PSD - densidade espectral de potência

ms - milisegundos

$\mathrm{CI}$ - contato inicial

TO - perda de contato do hálux com o solo

HPA - high pass algorithm

FVA - foot velocity algotithm

QTM - Qualisys Track Manager

$\mathrm{mV} / \mathrm{g}$ - milivolts por gravidade

A/D - analógico-digital

TAP - taxa de aceitação de peso

IA - índice de Amortecimento

EVA - Etileno acetato de vinila

SBR - Borracha de Butadieno Estireno (borracha sintética)

$\mathrm{NR}$ - Borracha natural 
Se os conhecimentos científicos e tecnológicos, atualmente disponíveis no mundo, fossem devidamente difundidos, adaptados e aplicados, contribuiriam para reduzir grande parte das inúmeras mazelas, que tanto nos entristecem $e$ envergonham como seres humanos.

Itiro Iida 